\title{
Performance of X-Ray Speckle Tracking Phase Retrieval Algorithms Towards a Dose Improvement.
}

\author{
$\underline{\text { Helene Labriet }}^{1,2}$, Emmanuel Brun ${ }^{2}$, Barbara Fayard ${ }^{1}$, Sylvain Bohic ${ }^{2}$, Sébastien Berujon ${ }^{3}$. \\ 1. Novitom, 3 avenue du Doyen Louis Weil 38000 Grenoble, France. \\ 2. Université Grenoble Alpes, Rayonnement Synchrotron et Recherche Médicale, 71 avenue des Martyrs \\ 38000 Grenoble, France. \\ 3. European Synchrotron Radiation Facility, 71 avenue des Martyrs 38000 Grenoble, France. \\ * Corresponding author, helene.labriet@novitom.com
}

X-ray Phase-Contrast Imaging methods (PCI) have seen rapid developments in the last decades due to their superior performances for imaging low-density objects and their ability to provide complementary information compared to traditional attenuation-based imaging. Since most of these methods require coherent beams, PCI is still today mainly performed at synchrotrons. Thus, significant resources are deployed by the community for the transfer of PCI techniques from synchrotrons to conventional x-ray sources with high divergence[1][2]. A recently introduced concept named X-ray Speckle Based Imaging (SBI) forms a new class of X-ray PCI techniques, sensitive to the first derivative of the phase. SBI presents many advantages including its experimental simplicity. In SBI the experimental complexity is translated to the numerical processing side which is complex and longer. Many developments are then focusing onto the processing algorithms in order to optimize the image quality but not always on dose reduction. SBI has already shown potential to provide both high sensitivity and high resolution imaging letting hope for a transfer on conventional X-ray sources at moderate efforts[3][1]. As a milestone towards the application of SBI at laboratory sources at low dose, further work is necessary regarding the optimization of the acquisition scheme and phase retrieval method. This study aims at defining the optimal strategy for acquisition and the associated phase retrieval methods for the transfer of the technique onto divergent polychromatic X-ray beam, at doses compatible with medical imaging.

Briefly, the SBI principle is to measure the displacement of intensity speckle patterns caused by a sample. To do so, we introduce a membrane that modulates spatially the beam intensity and then correlates pixelwise pairs of images taken with and without the sample. Here, we applied speckle based imaging using parallel beam on homemade phantoms composed of nylon fibers and polymers spheres and insect samples in order to image a sample with low density variation. We compared three different speckle tracking approaches exploring the effects of 2 different acquisitions schemes and 3 phase retrieval methods. The speckle vector tracking and its version with enlarged vectors (XSVT)[4] and the very much related Unified Modulated Pattern Analysis approach (UMPA)[5] use the same acquisition scheme but a different phase retrieval, minimization of correlation for UMPA and maximization of correlation for XSVT. XSVT and UMPA use a standard acquisition scheme with couples of images acquired with (IS) and without sample (IR). This scheme is repeated several times in order to get better statistics and reconstruct the phase and the dark-field maps. The x-ray speckle scanning (XSS) method[3] uses a little more complex acquisition scheme and an improved phase retrieval compared to XSVT. XSS method lies in a large number of acquisitions of the membrane and a moderate number of exposure of the samples. In other words XSS method uses an oversampling acquisition of reference to obtain a more resolved mesh scan of the diffusor, in our study a total of 49 references images were acquired with a regular displacement around each sample positions. Moreover, the XSS phase retrieval 
proposed here include the consideration of information in neighboring pixel to reduce the number of sample images required and to reduce the noise. As a result, for each pixel $\mathrm{P}$, two data arrays of 25 $\operatorname{IS}(\mathrm{P}, \tau)$ and $25 \operatorname{IR}(\mathrm{P}, \tau)$ were acquired and we obtained a set of $25 \operatorname{IS}(\mathrm{P}, \tau)$ and $1225 \operatorname{IR}(\mathrm{P}, \tau)$ for the XSS method. The experimental campaigns were conducted at the European Synchrotron Radiation Facility with a highly monochromatic and quasi-parallel $\mathrm{x}$-ray beam with a resulting pixel size of $3.5 \mu \mathrm{m}$. The membranes necessary for the generation of the speckle pattern were placed on a piezo xy-translation stage and are the same for all the acquisition schemes. The results from the different SBI methods were evaluated with three image quality metrics: structural similarity (SSIM), noise power spectrum and resolution.

If the image quality obtained with the different methods is comparable when using a high number of point images on sample, the XSS method shows its superiority when the number of sample exposures is reduced. Our comparison shows that the XSS method offers the highest potential in terms of dose reduction while maintaining a good image quality (Fig.1). Analysis of the SSIM shows that, when the dose is reduced by a factor 5, the overall loss of image quality is only reduced by a factor of 2 with XSS vs. a factor of 3.5 with UMPA and XSVT. Thanks to its low dose potential, we assume that XSS will permit to image sensitive medical samples.

We expect that XSS could allow to a low dose PCI acquisition on every sources type. We tested a second setup using a divergent beam obtained by creating a secondary source from the focalization of a Kirkpatrick-Baez (KB) mirror system (resulting pixel size: $0.16 \mu \mathrm{m}$ ) [6]. We reconstructed absorption, dark-field, phase contrast, and the two orthogonal differential phase maps from XSS combined with the highly diverging beam. The images are promising and show good results in terms of dose reduction compared to XSVT and UMPA. In this context, works were also initiated for the transfer of XSS scheme to setups based on laboratory sources. Further expansions of the XSS technique are also envisaged for tomographic reconstructions. . In conclusion, XSS is opening great opportunities for phase contrast imaging on laboratory X-ray sources with an emphasis on pre-clinical depiction as well as material science investigations.

References:

[1] H. Wang, Y. Kashyap, and K. Sawhney, Sci. Rep., vol. 6, p. 20476, Jan. 2016.

[2] T. Zhou et al., Opt. Lett., vol. 40, no. 12, p. 2822, Jun. 2015.

[3] S. Berujon and E. Ziegler, Phys. Rev. A, vol. 95, no. 6, p. 63822, Jun. 2017.

[4] S. Berujon and E. Ziegler, arXiv,2016.

[5] M.-C. Zdora et al., Phys. Rev. Lett., vol. 118, no. 20, p. 203903, May 2017.

[6] S. Berujon et al., Opt. Express, vol. 22, no. 6, pp. 6438-46, Mar. 2014.

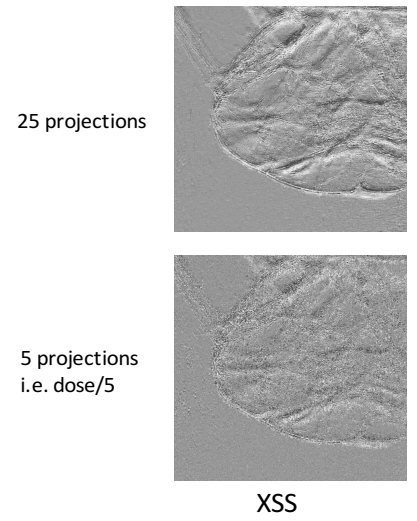

XSS
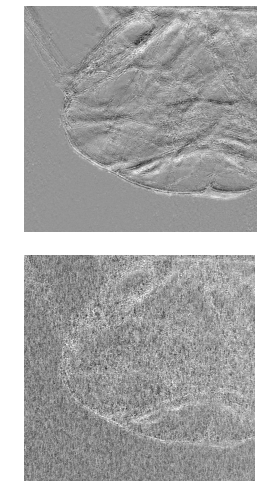

XSVT
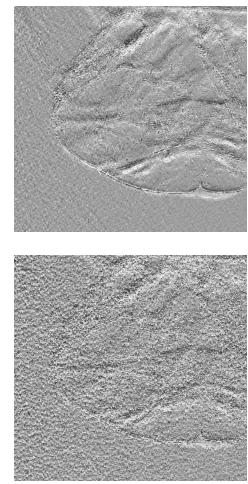

UMPA
Figure. 1. XY differential phase contrast map obtained with XSS, XSVT and UMPA method of a fly phantom using a parallel beam at $3.5 \mu \mathrm{m}$ and reconstructed with 25 points or 5 points on the sample. 